\title{
All-atom molecular dynamics simulations of multiphase segregated polyurea under quasistatic uniaxial compression
}

\author{
S. Heyden ${ }^{\mathrm{a}, *}$, M. Ortiz ${ }^{\mathrm{a}}$, A. Fortunelli ${ }^{\mathrm{b}}$ \\ ${ }^{a}$ California Institute of Technology, 1200 E California Blvd, Pasadena CA 91125, USA \\ ${ }^{b}$ CNR-ICCOM, Consiglio Nazionale delle Ricerche, via Giuseppe Moruzzi 1, 56124 Pisa, Italy
}

\begin{abstract}
We present an approach to model the mechanical response of multiphase segregated polyurea under large quasistatic uniaxial compression. The approach is based on a two-step procedure. We first conduct all-atom NVE molecular dynamics simulations of the fully-mixed and hard phases of polyurea. We then put forth a model of hard-phase activation based on Taylor, or meanfield, averaging and compute the composite response. The predictions of the present approach, with or without Taylor averaging, show remarkable agreement with data from plateimpact experiments.
\end{abstract}

Keywords: All-atom molecular dynamics, microphase segregated polyurea, hard phase activation

\section{Introduction}

Polyurea is an elastomer obtained by reaction of an isocyanate component and a synthetic resin blend component through step-growth polymerization. Polyurea chains are composed of long, flexible, aliphatic segments and short, rigid, aromatic segments (Yilgör et al., 2014; Agrawal et al., 2014; Yildirim, 2016), Figure 1 . The high polarity of hydrogen and oxygen atoms in urea groups promotes hydrogen bonding between urea linkages, which synergically combines with $\pi$ $\pi$ stacking interactions among aromatic rings (Sinnokrot and Sherill, 2004) and

\footnotetext{
*Corresponding author

Email addresses: heyden@caltech.edu (S. Heyden), ortiz@caltech. edu (M. Ortiz), alessandro. fortunelli@enr.it (A. Fortunelli)
} 
in turn leads to segregation into microphases consisting of hard chain segments and urea groups. The resulting copolymer displays a structured morphology of hard domains characterized by a high glass transition temperature $T_{g}$ embedded in a soft, viscoelastic matrix of low $T_{g}$ (Grujicic et al., 2011; Gamonpilas and McCuiston, 2012). This unique composition allows for two key mechanisms during deformation. Firstly, polyurea chains can possess hard segments belonging to different hard domains. During deformation, connecting soft segments allow hard domains to remain covalently linked to each other. The resulting coupling enables hard domains to undergo resonant motion during shock propagation, resulting in enhanced dissipation (Oswald et al., 2015). Secondly, the reversible breakage of $\pi-\pi$ interactions and urea hydrogen bonds within self-organized domains gives rise to remarkable self-healing properties that render polyurea attractive e.g. as a high-performance elastomeric biomaterial (Liu et al., 2012). The effect of variations in the soft/hard segment ratio on mechanical properties has been investigated by Fragiadakis et al. (2009) finding similar glass transitions and local segmental relaxation dynamics between materials possessing different segmental ratios. Furthermore, the materials' resistance to ballistic penetration is not affected by stoichiometry changes, suggesting that segmental dynamics rather than conventional mechanical properties determine the elastomers impact performance. In general, polyurea possesses many desirable material properties, such as high damping and dissipation as well as remarkable shear strength under pressure, reaching up to $500 \mathrm{MPa}$ at pressures of $9 \mathrm{GPa}$ (Jiao and Clifton, 2014), resulting in a broad range of applications, from lightweight monolithic coatings in translucent armor applications (Albrecht et al., 2013) to biomedical applications such as polyurea-nanoencapsulated aerogels for implantable devices (Yin et al., 2010).

Experimental investigations have revealed that microphase segregated areas in polyurea are of ribbon shape, with a characteristic diameter around $10 \mathrm{~nm}$ and an aspect ratio of 20:1 or greater (Grujicic et al., 2013a) as illustrated in Figure 2. Furthermore, the longest relaxation mode in polyurea exceeds tens of microseconds (Agrawal et al., 2014). Thus, atomistic molecular dynamics simulations, generally limited to simulation times of a few nanoseconds and systems which are a few nanometers in size, can only be used to investigate localized dynamics on short time scales. To overcome this limitation, a number of computational models have been put forth in the literature using coarse-grained molecular-level analyses (Grujicic et al., 2013b; Agrawal et al., 2014; Oswald et al., 2015). In Agrawal et al. (2014), the phenylmethaneaminobenzoate and tetramethylene-oxide units are treated as individual coarse-grained beads representing the collective degrees of freedom of the constituent atoms, with the parameters of the coarse-grained 


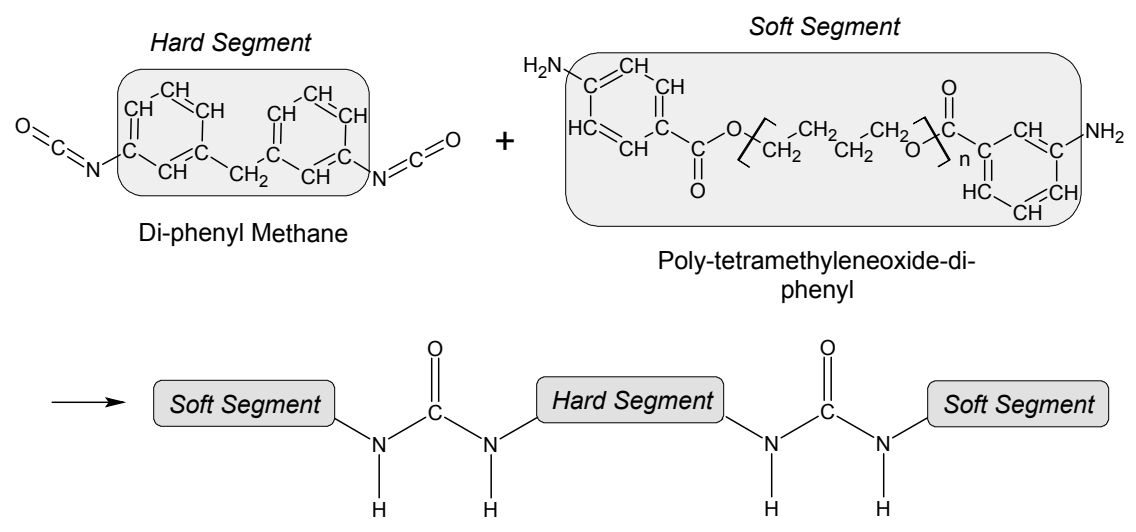

Figure 1: Formation of a polyurea molecule via reaction of an isocyanate component and a synthetic resin blend.

model obtained by applying a Boltzmann inversion approach based on structural distributions from fully atomistic simulations. Grujicic et al. (2013b) have applied a similar coarse-graining approach, whereby particular emphasis is placed on the shock-mitigation potential of polyurea and its underlying mechanisms, finding that polyurea's high ability of shock wave attenuation and dispersion likely results from shock-induced crystallization of hard domains and coordinated lateral motion of soft domains. While the approach of treating functional units within the polyurea chains as coarse-grained beads decreases computational cost and thus extends the realizable domain sizes and simulation times, its main drawback is the introduction of additional model parameters in order to characterize the interactions between individual beads.

In this work, we adopt an alternative strategy structured in two steps: Firstly, we conduct all-atom molecular dynamics (MD) simulations which aim at characterizing material properties of the individual phases of polyurea. Then, we develop a model of hard-phase activation and derive the composite material behavior by recourse to Taylor, or meanfield, averaging. It bears emphasis that this approach is based solely on interatomic potentials and requires no additional empirical parameters. A comparison between the behavior of the composite model and that of the fully-mixed state of polyurea evinces the influence of multiphase segregation in polyurea under quasistatic uniaxial compression. The composite model is validated against plate-impact experiments as performed by Jiao and Clifton (2014). 


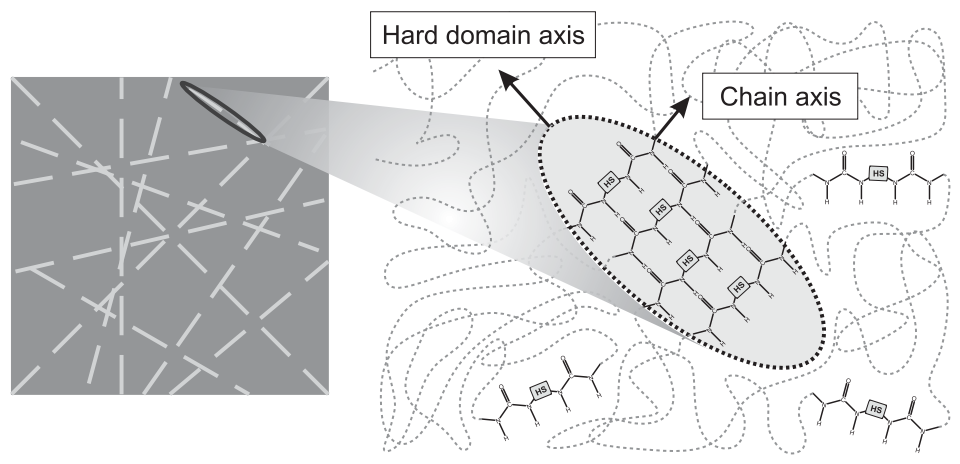

Figure 2: Schematic of hard domain structure within the fully-mixed matrix in the undeformed configuration with hard segment alignment as presented in Grujicic et al. (2015).

\section{Model development}

Our model of microphase-segregated polyurea includes both atomistic and continuum level contributions. At the atomistic scale, we generate and analyze different representative volume elements to represent the individual phases of the material (Section 2.1). Based on the atomistic description, we then put forth a model of hard-phase activation giving the behavior of structured polyurea under uniaxial deformation on the continuum scale (Section 2.2).

\subsection{Atomistic model}

We construct three different representative volume elements (RVEs) with a total of 5 samples generated for each type of RVE in order to take statistical variations into account:

- RVE (Type I) is tailored towards a representation of the fully-mixed state of polyurea (i.e., without taking microphase segregation into account), Figures 3,5

- RVE (Type II) is constructed so as to represent the hard phase of polyurea (as depicted in Figure 7)

- RVE (Type III) is tailored towards a representation of the soft matrix of polyurea (i.e., the fully-mixed state of Type I with a reduced amount of hard phase segments), Figure 6 
RVE (Type I) is used to estimate the influence of microphase segregation by comparison to the composite material response and is constructed as follows. The weight percent of hard segments in Polyurea 1000 is on the order of $36 \%$ (Rinaldi et al., 2011). To achieve this composition, we replicate a single polyurea molecule via a copy-and-attach process into a ten-fold polyurea chain with a total of 2422 atoms. Thereby, single polyurea molecules are tailored towards a representation of P1000 with an approximate molecular weight of 1000 a.m.u. per soft segment. For this rendition of polyurea, $n=14$ repeat units are used per soft segment as shown in Figure 1. Following static minimization, chains are used as building blocks to populate a cubic simulation cell of size $5 \mathrm{~nm}$ containing a total of 14,532 atoms, Figure 3. We relax the resulting RVE in an NPT ensemble at ambient conditions for $10 \mathrm{~ns}$ using the molecular dynamics software LAMMPS (Plimpton, 1995) to achieve a target equilibrium density of $1025.09 \mathrm{~kg} / \mathrm{m}^{3}$. Following Agrawal et al. (2014), in order to generate an amorphous structure we subject the RVE to an annealing process consisting of a first relaxation in an NPT ensemble at $T=$ $500 \mathrm{~K}$ and $p=1 \mathrm{~atm}$ for $8 \mathrm{~ns}$, followed by quenching to $T=300 \mathrm{~K}$ for $8 \mathrm{~ns}$ and a final relaxation at ambient conditions for $16 \mathrm{~ns}$, see Figures 3 and 5. The annealing temperature and time are sufficient to melt the RVEs, thus eliminating possible artifacts due to the initial ordered structure. To ensure that the system has reached a fully-equilibrated state after the final relaxation step, we check that there is no drift in the total energy trajectory of the system.

Density profiles along each RVE dimension, whereby a spacing of ten bins for each dimension was chosen, furthermore show that the annealing procedure decreases the range of variations between densities in each direction. As illustrated in Figure 4, the largest difference in atom densities before the annealing procedure is applied, is located in bin 10 with a value of $0.9 \%$ between directions $\mathrm{x}$ and $\mathrm{z}$. After the annealing procedure is applied, the largest variation can be found in bin 3 with a difference of $0.575 \%$ in atom density between directions $\mathrm{x}$ and $y$. These fluctuations are minor and expected at the nanometer level for an amorphous material.

The generation of samples of the soft matrix RVE (Type III) follows a similar protocol. Based on a tapping-mode atomic force micrograph presented in Grujicic et al. (2013a) and by recourse to binary pixel analysis, we determine the fraction of multiphase segregated hard domains to be $\alpha_{H}=0.2326 \equiv 23.26 \%$. From this fraction, we infer the number of randomly dispersed hard segments from the 10 -fold polyurea molecules and subject the corresponding representative volume elements to the relaxation and annealing procedure previously outlined for the generation of fully-mixed RVEs (Type I). 


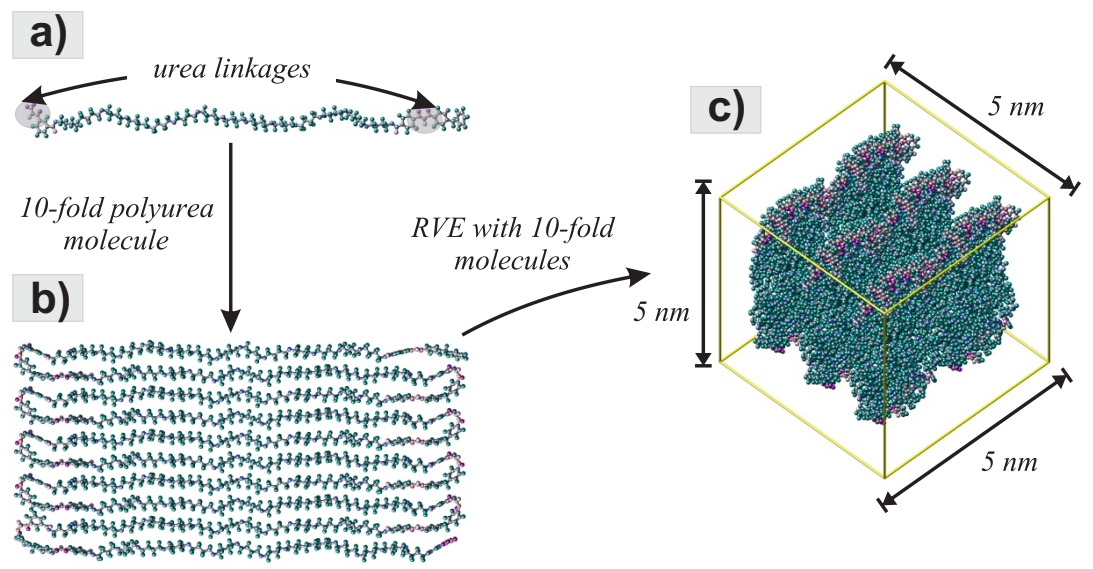

Figure 3: Generation of a representative volume element of fully-mixed polyurea (Type I). a) Single polyurea chain. b) 10-fold polyurea molecule grown via copy-and-attach-process. c) RVE of the initial configuration of fully-mixed polyurea (15k atoms) before annealing created from population of a cubic cell with polyurea chains.

The generation of hard phase RVEs (Type II) proceeds via a biased global optimization procedure applied to different representative volume elements with 8 monomers composed of hard segments and urea linkages terminated by methyl benzoate $\left(\mathrm{C}_{5} \mathrm{H}_{5} \mathrm{CO}_{2} \mathrm{CH}_{3}\right)$. We then put such hard-phase RVEs through prolonged relaxation runs of $20 \mathrm{~ns}$ at ambient conditions, (instead of the relaxation and annealing procedure applied to RVEs of Type I and Type III), in order to promote the formation of hydrogen bonds and finally select the so derived minimum-energy configuration as representative RVEs as depicted in Figure 7.

For all different types of RVEs, bonded and non-bonded interactions between atoms that constitute the polyurea chains are modeled using the OPLSAA force field (Jorgensen and Tirado-Rives, 1988; Jorgensen et al., 1995), which is adequate to describe the given polymeric ensemble (Mondal et al., 2013). With respect to non-bonded interactions, Pauli repulsion and van-der-Waals forces are taken into account via a Lennard-Jones potential according to

$$
E_{L J}=4 \epsilon\left[\left(\frac{\sigma}{r}\right)^{12}-\left(\frac{\sigma}{r}\right)^{6}\right]
$$

with cut-off $r<r_{c}$. In addition, coulombic interactions are taken into account as

$$
E_{\text {Coul }}=\frac{C q_{i} q_{j}}{\epsilon r_{i j}}
$$



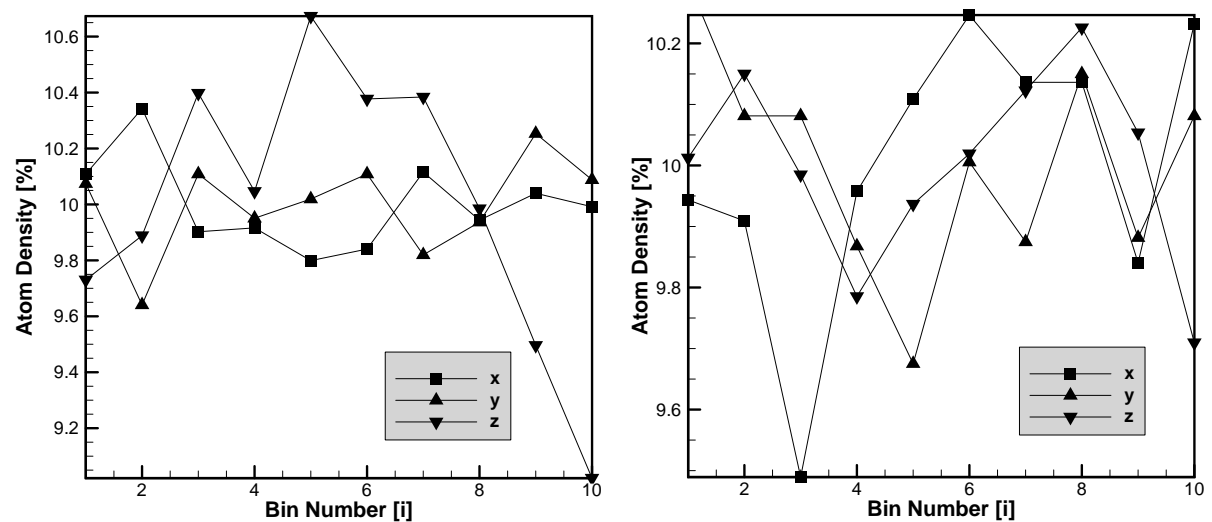

Figure 4: Density profiles along each RVE dimension before (left) and after the annealing procedure is applied (right).

whereby long-range electrostatic interactions are evaluated by recourse to the Ewald summation algorithm. Here, we employ a global cut-off $r_{c}=10 \AA$ and an accuracy of $\delta \mathbf{f}=10^{-4} \mathrm{Kcal} /(\mathrm{mol} \AA)$ in the desired relative error in forces in the calculation of long-range Coulomb interactions. It bears mentioning that by default, non-bonded interactions within LAMMPS are taken into account for atoms which are four or more bonds apart, whereas the OPLSAA force field also includes 1,3 interactions. This inconsistency can be eliminated by including appropriate special bonds within the LAMMPS modeling framework. For bonded interactions between atoms, harmonic functions for both covalent bonds and angular terms are used according to

$$
\begin{aligned}
& E_{\text {Bond }}=K_{\text {Bond }}\left(r-r_{0}\right)^{2} \text { and } \\
& E_{\text {Angle }}=K_{\text {Angle }}\left(\theta-\theta_{0}\right)^{2} .
\end{aligned}
$$

Finally, a Fourier potential is used to model torsional terms (with $n_{i} \geq 0$ and $m=3)$ as

$$
E_{\text {Torsion }}=\sum_{i=1, m} K_{i, \text { Torsion }}\left[1+\cos \left(n_{i} \Phi-d_{i}\right)\right] .
$$

As a verification test of the adequacy of the proposed force field for purposes of simulating polyurea, we use a system containing functional urea groups of known configuration. Thereby, we reproduce the crystalline structure of $N, N^{\prime}$ bis(2,3-bis(benzoyloxymethyl)-4-(methoxycarbonyl)cyclopentyl)urea as published in the Cambridge Crystallographic Database (Allen, 2002; Figueira et al., 
a)

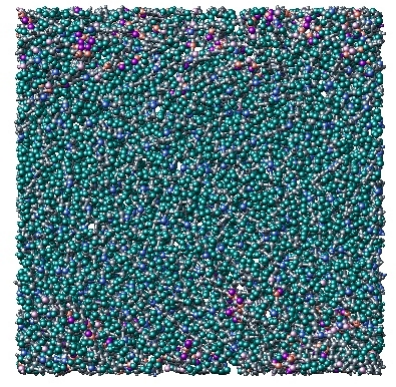

b)

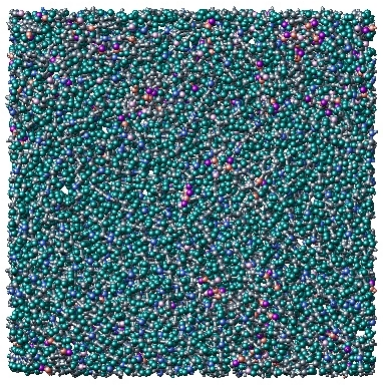

Figure 5: a) RVE (Type I) after relaxation of Figure 3c) at ambient conditions showing a distribution of urea linkages highly dependent on the initial configuration of polyurea chains. b) RVE (Type I) after the annealing procedure showing a fully-mixed distribution of urea linkages within the computational cell.

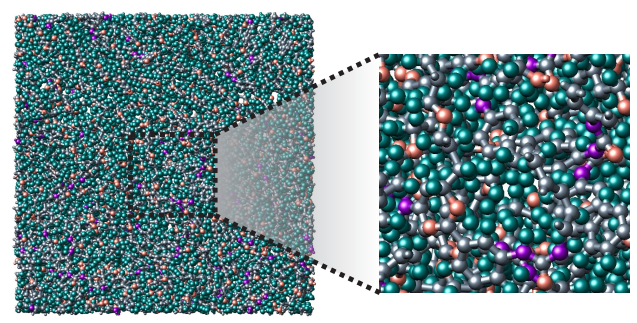

Figure 6: Soft domain RVE (Type III) after annealing with close-up view of the atomistic structure.

2002; Groom et al., 2016). Specifically, we recover the crystalline density with an accuracy of $92.71 \%$. In addition, we reproduce hydrogen bond lengths between functional urea groups with an accuracy of $98.97 \%$. For a further validation of the proposed force field, we calculate the specific heat capacity at constant volume by considering the instantaneous fluctuations in the energy as

$$
C_{V}=\frac{\left\langle E^{2}\right\rangle-\langle E\rangle^{2}}{k_{B} T}=\frac{\left\langle(E-\langle E\rangle)^{2}\right\rangle}{k_{B} T}=\frac{\operatorname{Var}(E)}{k_{B} T},
$$

whereby $E=E_{P o t}+E_{K i n}$ (Leach, 2001). Similar to calculations performed in the following, $C_{V}$ is first calculated for a total of five samples of each hardphase and soft-matrix systems, from which a mean value is derived by recourse to Taylor averaging. Applying a rule of mixtures as outlined in Section 2.2 then gives a value of $3.9 \mathrm{~J} /\left(\mathrm{cm}^{3} \mathrm{~K}\right)$ for the composite specific heat capacity at constant 


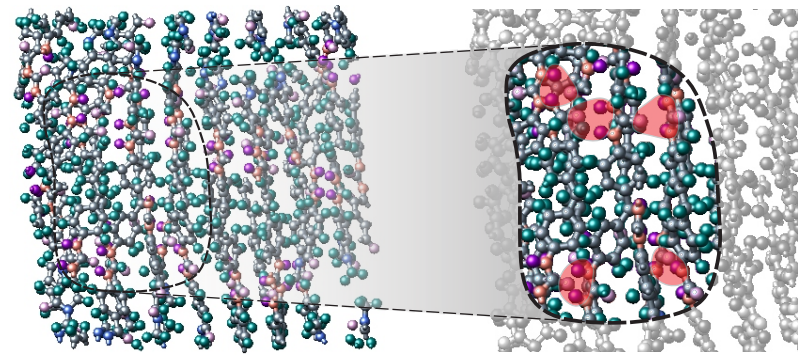

Figure 7: Hard domain RVE (Type II) after relaxation with a close-up view including highlighted regions denoting H-Bonds between urea linkages.

volume, which agrees well with previously reported values for the same degree of polymerization of the polyurea chains (Agrawal et al., 2014).

\subsection{Composite model}

We propose a model of hard-phase activation based on Taylor averaging as a means of deriving the composite material response from the response of its matrix and hard phase constituents. The Taylor, or Voigt, assumption (Voigt, 1889) implies that a uniform affine deformation applied to a representative volume element of multiphase segregated polyurea equals the deformation experienced by the individual phases within the volume element. This assumption allows us to calculate the material response of matrix and hard-phase RVEs separately, and to subsequently derive the composite material response from the rule of mixtures

$$
P_{x x, \text { composite }}=\left(1-\alpha_{H}\right) P_{x x, \text { Soft }}+\alpha_{H} P_{x x, \text { Hard }},
$$

whereby $P_{x x}$ is the first Piola-Kirchhoff stress component, or force per unit undeformed area, in the direction of compression. Furthermore, $\alpha_{H}$ denotes the mixing parameter as introduced in Section 2.1. For present purposes, the affine deformation of interest is a uniform upsetting of the RVE in the direction of compression. Specifically, if $x$ is the initial coordinate of a material point in the direction of compression and $y$ is the corresponding component in the deformed configuration, then $y=\lambda x$, with $\lambda \leq 1$ the compression ratio, for all material points in the RVE, cf. Figure 8.

Before Equation (6) can be applied to calculate the composite material response, we need to find an estimate for the stresses within the hard domains $P_{x x, \text { Hard }}$. In contrast to the soft matrix, hard phase RVEs show a marked anisotropy 


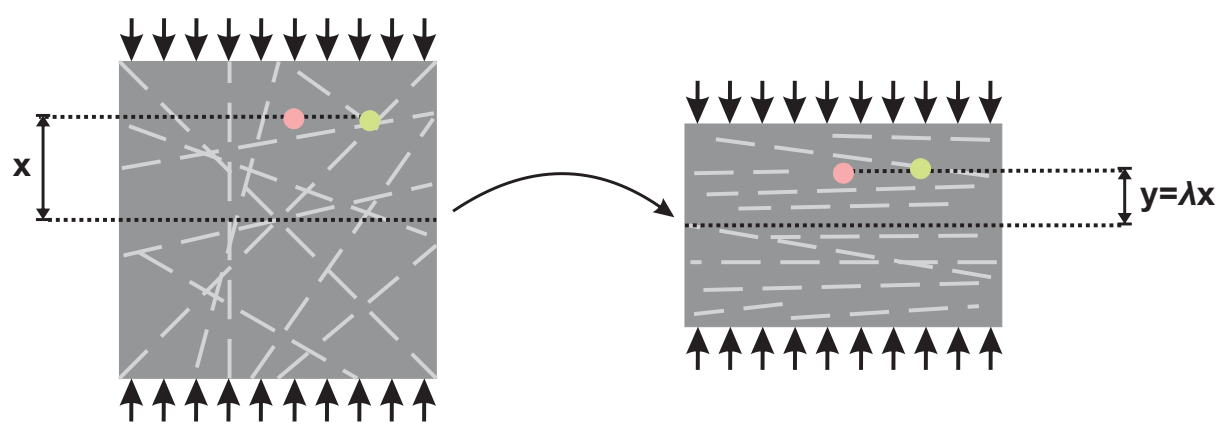

Figure 8: Under the Voigt assumption, the deformation state imposed on a multiphase segregated volume element equals the deformation experienced by the individual phases. Material points within the soft matrix (pink) and material points in the hard phases (green) therefore deform identically.

due to their structured morphology consisting of $\pi-\pi$ stacking interactions and hydrogen bonds along the hard domain axis and covalent bonds along the chain axis, cf. Figure 2. Using molecular dynamics simulations, the stress-strain relation under compression along each of these axes can be computed. To find the resulting stress-strain behavior for a randomly oriented chain, we proceed as follows.

For an isotropic distribution of randomly-oriented hard domains in the undeformed configuration, the overall strain energy density of hard domains is assumed to be of the form

$$
W_{\text {Hard }}(\mathbf{F})=\int_{\mathcal{S}}\left(W_{\|}\left(\lambda_{\|}\right)+W_{\perp}\left(\lambda_{\perp}\right)\right) d S,
$$

which corresponds to an assumption of transverse isotropy, vanishing Poisson effect, and to the additional assumption that the transverse shear deformation of the hard domains is fully relaxed. Here, $\mathbf{F}=\{\{1,0,0\},\{0, \lambda, 0\},\{0,0,1\}\}$ denotes the deformation gradient which describes the compression of the RVE, and $\lambda_{\|}$ and $\lambda_{\perp}$ are the stretches along the hard domain and chain axes, respectively. Furthermore, Equation (7) is integrated over the unit sphere $d S$ in order to account for energy contributions from all possible hard phase orientations. We find the stretches along the hard phase axis $\mathbf{N}_{\|}$and the chain axis $\mathbf{N}_{\perp}$ from the kinematical relations

$$
\begin{aligned}
\lambda_{\|} & =\sqrt{\mathbf{N}_{\|} \cdot\left(\mathbf{F}^{T} \mathbf{F}\right) \mathbf{N}_{\|}} \text {and } \\
\lambda_{\perp} & =\sqrt{\mathbf{N}_{\perp} \cdot\left(\mathbf{F}^{T} \mathbf{F}\right) \mathbf{N}_{\perp}} .
\end{aligned}
$$


Using these relations, the first Piola-Kirchhoff stress tensor of hard domains can be calculated as

$$
\mathbf{P}_{\text {Hard }}(\mathbf{F})=\frac{\partial W_{\text {Hard }}(\mathbf{F})}{\partial \mathbf{F}}
$$

A notable effect of this ansatz is that the hard domains, which are randomly oriented initially, rotate during deformation and become increasingly parallel to the plane normal to the compression direction.

\section{Results and Discussion}

To summarize the computational approach: Molecular Dynamics (MD) simulations of representative volume elements (RVEs) of the soft matrix, hard phases and fully-mixed state of polyurea under uniaxial compression are first carried out, Section 3.1; the composite material response is then calculated from separate simulations of soft matrix and hard phase RVEs and compared to simulations of the fully-mixed state of polyurea in order to investigate the influence of multiphase segregation under uniaxial compression. In Section 3.2, the results obtained through this approach are presented and validated against pressure-shear plate impact experiments on polyurea specimens.

\subsection{Uniaxial compression tests}

To investigate the influence of microphase segregation in polyurea under conditions of quasistatic uniaxial compression, we subject the individual RVEs to a displacement controlled uniaxial deformation. Each deformation step consists of a uniaxial compression of $1.25 \%$, followed by relaxation in an NVE ensemble for $1 \mathrm{~ns}$. Averages in the form of mean stress-strain curves are calculated from the response of 5 different samples for each type of RVE, namely, fully mixed, soft matrix and hard-phase systems.

The computational model will be validated against the pressure-shear plate impact experiments on polyurea specimens performed by Jiao and Clifton (2014). In these experiments, thin polyurea layers are cast on both flyer and anvil plates. Rear-surface velocities are measured and one dimensional elastic wave theory is used to obtain tractions and particle velocities at the flyer/anvil interface. It is of particular importance to note that the incident longitudinal wave arrives first at the rear surface and reverberates through the thickness of the sample until the stress becomes uniform. This fully-equilibrated normal stress $P_{x x}$ is used for comparison with molecular dynamics simulations. It can thus be concluded that the uniaxial strain compression simulations performed through a sequence of equilibration 
steps adequately represent the experiments. Furthermore, the experimental setup allows for changes in temperature within the sample during the impact phase, which immediately suggests a comparison with molecular dynamics simulations in an NVE ensemble.

Results for the fully-mixed state of polyurea and the soft matrix case are shown in Figure 9. RVEs of fully-mixed polyurea under uniaxial compression show a remarkably good agreement with experiment, notwithstanding a slight over- and underestimation of the stress in the medium- and high compression regimes, respectively. We note that, owing to its particular molecular structure, Figure 2, the stress-strain response of the hard phase may be expected to be markedly anisotropic. Indeed, as shown in Figure 10, the stress-strain behavior is strongly dependent on the loading direction. Specifically, the response in uniaxial compression along the chain axis, which is perpendicular to the hard domain axis, is stiffer than the response in uniaxial compression along the hard domain axis, which is the result of hydrogen and $\pi-\pi$ bonding. As expected, the response of the soft matrix is characterized by lower stress levels than the hard-phase response. Since simulations are conducted in an NVE ensemble, they additionally predict the increase in temperature during deformation. This rise in temperature is expected to occur in the pressure-shear plate impact experiments due to reverberating pressure waves within the material. In fully-mixed samples the simulations consistently predict a temperature increase of about $150 \mathrm{~K}$ at a compression ratio of $\lambda=0.6$, see Figure 11. Thereby, the temperature follows from the kinetic energy of the system as

$$
\sum_{i=1}^{N} \frac{1}{2} m_{i}\left|\mathbf{v}_{i}\right|^{2}=\frac{3}{2} N k_{B} T
$$

where $N, k_{B}$ and $T$ denote the total number of atoms in the system, the Boltzmann constant and the absolute temperature, respectively, $m_{i}$ is the atomic mass and $v_{i}$ denotes the atom's velocity.

\subsection{Composite material response}

The model of hard-phase activation proposed in Section 2.2, based on Taylor averaging as a means of deriving the composite material response from the response of its matrix and hard phase constituents, is applied to the individual responses obtained from molecular dynamics simulations as shown in Figure 10. The resulting stress-strain behavior of the composite is depicted in Figure 12. Owing to the stiffer transverse response of the hard domains, relative to their axial 


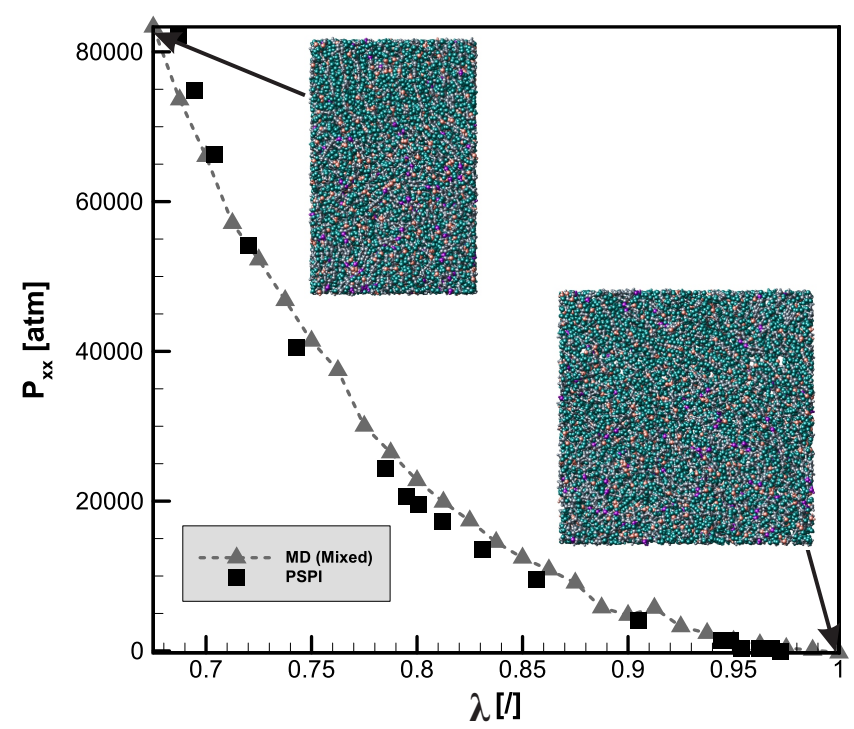

Figure 9: Fully mixed configuration of polyurea. Comparison of calculated and experimental Jiao and Clifton (2014) stress-strain response in uniaxial compression.

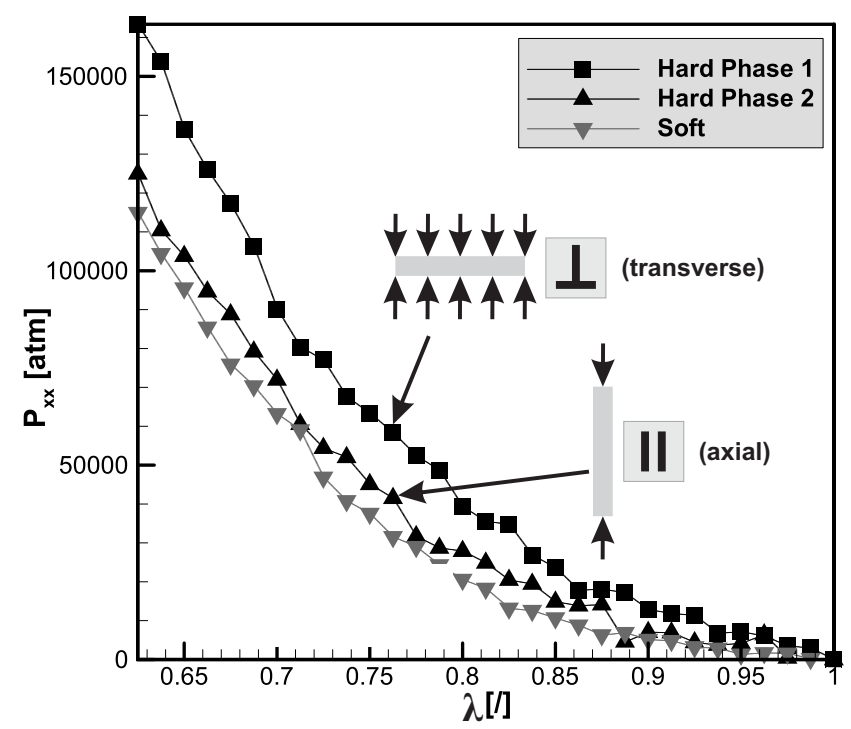

Figure 10: Hard and soft phases of polyurea. Calculated stress-strain responses in uniaxial compression. For the hard phase, the response is calculated for a loading axis in the axial $(\|)$ and transverse $(\perp)$ directions. 


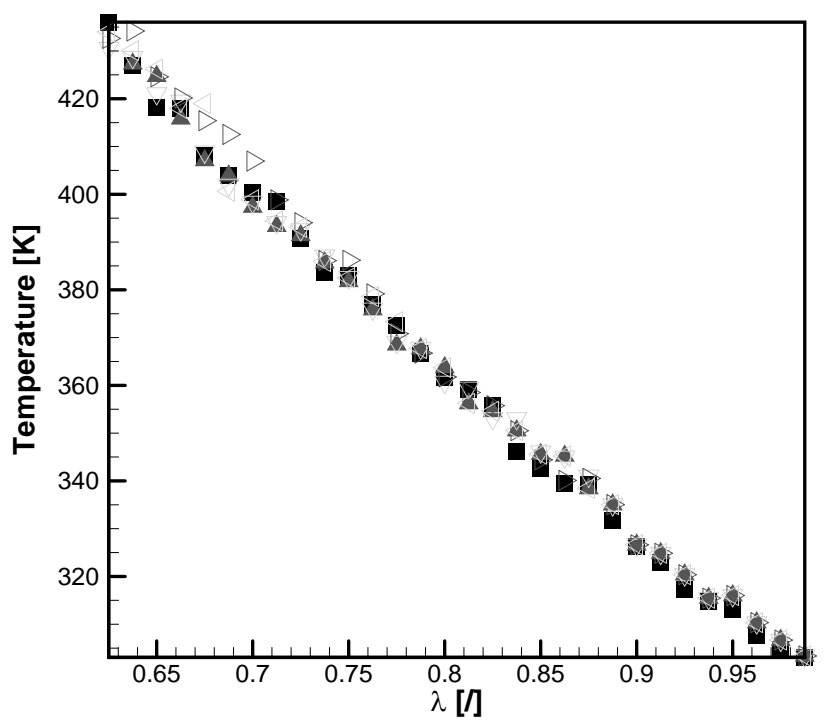

Figure 11: Fully mixed configuration of polyurea. Calculated temperature increase during uniaxial compression for five different samples.

response, the deformation-induced alignment of the hard domains tends to stiffen the overall response of the composite RVE, but this stiffening effect is small and the composite response remains ostensibly close to that of the fully-mixed phase, see Figure 9. Figure 13 furthermore depicts a sensitivity analysis of changes in the mixing parameter $\alpha_{H}$ and the corresponding effect on the composite material response. As can be noted, a decrease of $\alpha_{H}$ does not have a notable effect in the higher compression regime. A similar behavior can be observed for moderate increases of $\alpha_{H}$ ranging up to $\alpha_{H}=0.38$.

\subsection{Testing of the Taylor hypothesis}

The proposed composite model is based on the Taylor hypothesis, which is predictive for cases in which the contrast between individual phases is low. To assess the validity of the Taylor assumption for the case of multiphase segregated polyurea, this Section presents fully three dimensional unit cell calculations using the finite element method. Results are then compared to the composite material model outlined in Section 2.2, whereby contributions of the individual phases are approximated by third order polynomial functions.

The representative unit cell used in finite element simulations is illustrated in Figure 14. The volume of the ribbon-shaped inclusion is chosen corresponding 


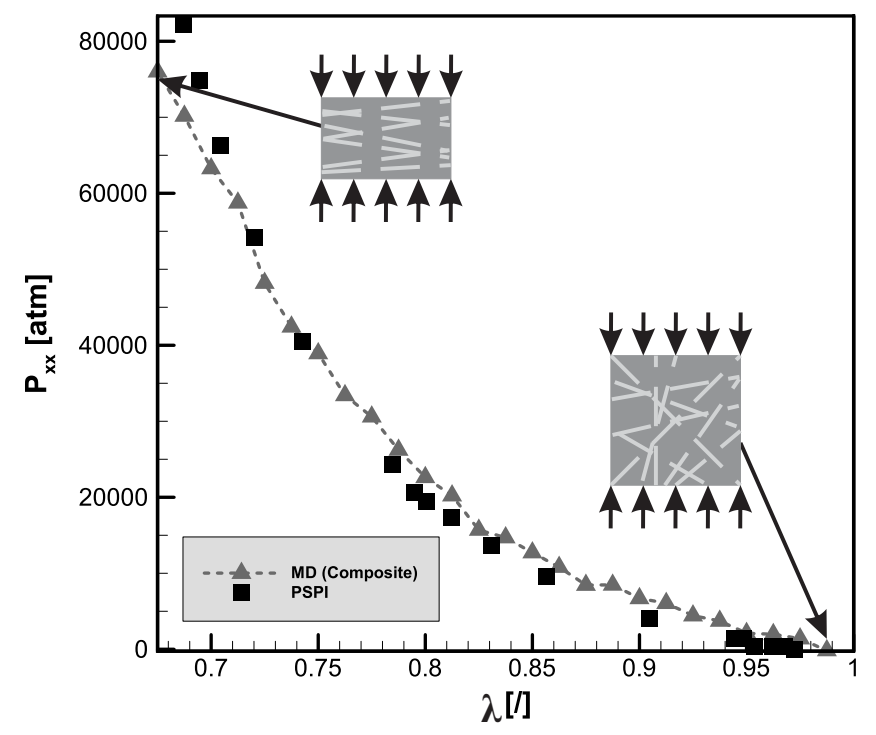

Figure 12: Composite model of polyurea. Comparison of calculated and experimental (Jiao and Clifton, 2014) stress-strain response in uniaxial compression. With increasing compression, hard domains tend to align perpendicular to the loading direction.

to the fraction of multiphase segregated hard domains of $\alpha_{H}=0.23$. For the material model used in calculations, we resort to the three-term Yeoh model as a prime candidate in modeling soft materials (Yeoh, 1993), whereby the strain energy density takes on the form

$$
W(\mathbf{F})=\kappa(J-1)^{2}+C_{10}\left(\bar{I}_{1}-3\right)+C_{20}\left(\bar{I}_{1}-3\right)^{2}+C_{30}\left(\bar{I}_{1}-3\right)^{3} .
$$

Here, $J=\operatorname{det}(\mathbf{F})$ and $\bar{I}_{1}=\operatorname{tr}(\mathbf{F}) / J^{2 / 3}$. Material parameters are then fitted to the individual phase responses obtained from molecular dynamics simulations as shown in Figure 10, from which fitting parameters follow as: $\kappa_{\text {matrix }}=1.01 \mathrm{GPa}$, $C_{10, \text { matrix }}=1.4 \mathrm{GPa}, C_{20, \text { matrix }}=9.2 \mathrm{GPa}, C_{30, \text { matrix }}=2 \cdot 10^{-9} \mathrm{GPa}, \kappa_{\text {domain }}=$ $1.0 \mathrm{GPa}, C_{10, \text { domain }}=3.2 \mathrm{GPa}, C_{20, \text { domain }}=6.1 \mathrm{GPa}, C_{30, \text { domain }}=10^{-10} \mathrm{GPa}$, $\kappa_{\text {chain }}=1.1 \mathrm{GPa}, C_{10, \text { chain }}=5.2 \mathrm{GPa}, C_{20, \text { chain }}=6.5 \mathrm{GPa}$, and $C_{30, \text { domain }}=$ $2 \cdot 10^{-9} \mathrm{GPa}$.

The unit cell is discretized into a total of 14322 linear tetrahedral elements and subsequently subjected to uniaxial compression along the hard phase domain axis, whereby affine dirichlet boundary conditions are applied. Displacement increments are set to $\Delta u=0.2 \%$ and the volumetric average of the first PiolaKirchhoff stress is calculated up to a total stretch of $30 \%$. It can be noted from 

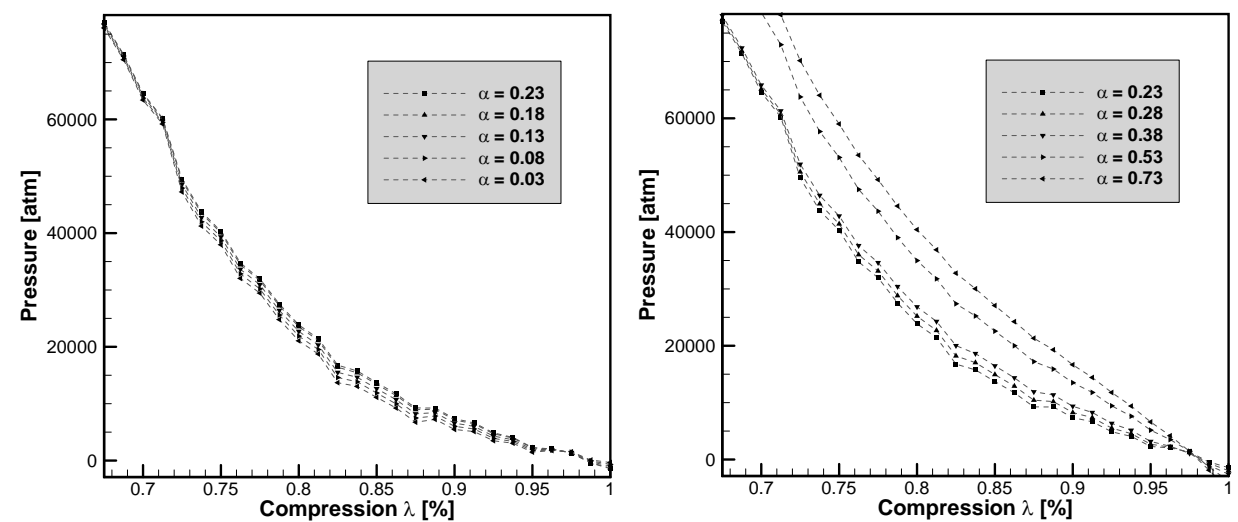

Figure 13: Sensitivity analysis of changes in the mixing parameter $\alpha_{H}$ and the corresponding effect on the composite material behvior.

Figure 14 that the composite material behavior based on the Taylor hypothesis shows good agreement with finite element calculations, with deviations within a $5 \%$ margin in the medium compression regime. The proposed hard phase activation model is therefore predictive in modeling the material behavior of multiphase segregated polyurea under conditions of quasistatic uniaxial compression.

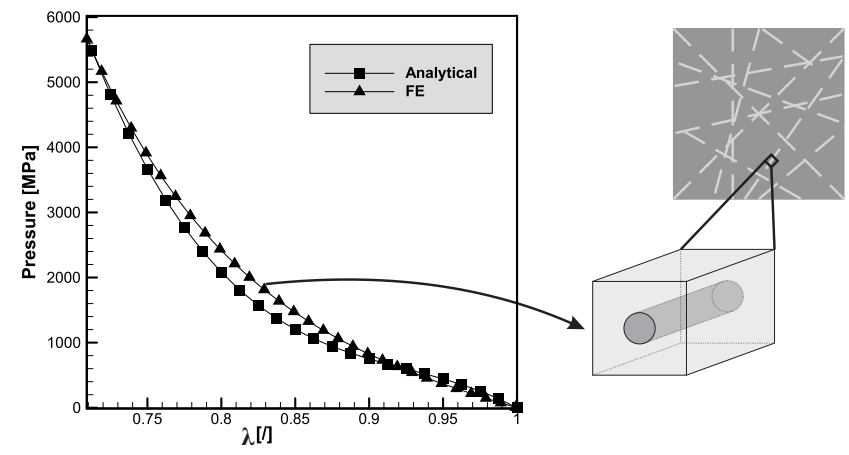

Figure 14: Comparison between the composite material model based on the Taylor assumption and finite element simulations. The inset shows the unit cell used in finite element simulations representing a volume element of soft matrix with an embedded ribbon-shaped domain. 


\subsection{Discussion}

We have presented a model of the mechanical response of multiphase segregated polyurea P1000 under quasistatic uniaxial compression. An investigation of suitable representative volume elements (RVEs) based on all-atom molecular dynamics simulations reveals differing individual responses of the soft matrix, the hard-phase domains, and the fully-mixed configuration. A simple Taylor averaging of the constituent phases then gives the behavior of the composite.

Particularly relevant to hard-phase activation is the observation that the hardphase RVEs exhibit anisotropic behavior. This anisotropy is attributable to the molecular structure of the hard phase, in which individual hard segments are solely connected via non-covalent bonds along the hard domain axis. While previous modeling efforts primarily focused on anisotropy due to the overall structure of hard phases embedded into a soft matrix, results presented in this work suggest that anisotropy within hard phases is an additional contributing factor underlying the material behavior of polyurea. The composite behavior derived from the hard-phase activation model agrees well with experiments over a broad range of compression. A comparison to fully three dimensional unit cell calculations using the finite element method furthermore shows that the Taylor assumption is valid for modeling multiphase segregated polyurea under conditions of quasistatic uniaxial compression.

Interestingly, we find that the composite response differs only slightly from that of the fully-mixed configuration, which also shows remarkable agreement with experiment. This finding suggests that, under conditions of quasistatic uniaxial loading, the segregation of polyurea hard chain segments into hard domains does not have an appreciable effect on the overall response of the composite. In contrast, multiphase segregation may be expected to exert a strong influence on the dynamic properties of polyurea, especially as regards dissipation during shock loading as suggested in Oswald et al. (2015); Grujicic et al. (2013b). This idea is consistent with the finding of Fragiadakis et al. (2009) that the impact performance of polyurea is governed by segmental dynamics rather than stoichiometry variations.

The hard-phase activation is susceptible to a number of extensions and enhancements in which more complex couplings between the hard-phases and the soft matrix are taken into account. Future modeling efforts can for instance include the transverse shear deformation within hard domains. Finally, the results presented in this study may be used as synthetic material data in order to inform and calibrate continuum level models and calculations. 


\section{Acknowledgements}

SH and MO gratefully acknowledge support from the Office of Naval Research through grant N00014-14-1-0296.

\section{References}

Agrawal, V., Arya, G., Oswald, J., 2014. Simultaneous iterative boltzmann inversion for coarse-graining of polyurea. Macromolecules 47, 3378-3389.

Albrecht, A. B., Liechti, K. M., Ravi-Chandar, K., 2013. Characterization of the transient response of polyurea. Experimental Mechanics 53, 113-122.

Allen, F. H., 2002. The cambridge structural database. Acta Cryst. B58, 380-388.

Figueira, M. J., Caamaño, O., Fernández, F., Blanco, J. M., 2002. Synthesis of ()-c-4-amino-r-1,t-2,c-3-cyclopentanetrimethanol and higher homologues of 8azapurine arabino-carbocyclic nucleosides. Tetrahedron 58, 72337240.

Fragiadakis, D., Gamache, R., Bogoslovov, R. B., Roland, C. M., 2009. Segmental dynamics of polyurea: Effect of stoichiometry. Polymer 51, 178-184.

Gamonpilas, C., McCuiston, R., 2012. A non-linear viscoelastic material constitutive model for polyurea. Polymer 53, 3655-3658.

Groom, C. R., Bruno, I. J., Lightfoot, M. P., Ward, S. C., 2016. The cambridge structural database. Acta Cryst. B72, 171-179.

Grujicic, M., Pandurangan, B., King, A. E., Runt, J., Tarter, J., Dillon, G., 2011. Multi-length scale modeling and analysis of microstructure evolution and mechanical properties in polyurea. J Mater Sci 46 (6), 1767-1779.

Grujicic, M., Snipes, J. S., Ramaswami, S., Galgalikar, R., Runt, J., Tarter, J., 2013a. Molecular- and domain-level microstructure-dependent material model for nano-segregated polyurea. Multidiscipline Modeling in Materials and Structures $9(4), 548-578$.

Grujicic, M., Snipes, J. S., Ramaswami, S., Yavari, R., Runt, J., Tarter, J., Dillon, G., 2013b. Coarse-grained molecular-level analysis of polyurea properties and shock-mitigation potential. Journal of Materials Engineering and Performance 22 (7), 1964-1981. 
Grujicic, M., Yavari, R., Snipes, J. S., Ramaswami, S., Jiao, T., Clifton, R. J., 2015. Experimental and computational study of the shearing resistance of polyurea at high pressures and high strain rates. J Mater Eng Perform 24, 778798.

Jiao, T., Clifton, R. J., 2014. Measurement of the response of an elastomer at pressures up to 9 gpa and shear-rates of $10^{5}-10^{6} \mathrm{~s}^{-1}$. Journal of Physics: Conference Series 500 (11), 1-6.

Jorgensen, W. L., Maxwell, D. S., Tirado-Rives, J., 1995. Development and testing of the opls all-atom force field on conformational energetics and properties of organic liquids. Journal of the American Chemical Society 118 (45), 112251123.

Jorgensen, W. L., Tirado-Rives, J., 1988. The opls force field for proteins. energy minimizations for crystals of cyclic peptides and crambin. Journal of the American Chemical Society 110 (6), 1657-1666.

Leach, A. R., 2001. Molecular Modeling: Principles and Applications, 2nd Edition. Pearson Education Limited, Harlow, England.

Liu, C.-C., Zhang, A.-Y., Feng, Z.-G., 2012. Self-healing biodegradable poly(urea-urethane) elastomers based on hydrogen bonding interactions. Chinese Journal of Polymer Science 31 (2), 251-262.

Mondal, J., Stirnemann, G., Berne, B. J., 2013. When does tmao fold a polymer chain and urea unfold it? J Phys Chem B 117 (29), 8723-8732.

Oswald, J., Arya, G., Cui, Z., Brinson, L. C., 2015. Molecular and coarsegrained methods for microstructure-property relations in hsrep. In: Roshdy, R. (Ed.), Elastomeric Polymers with High Rate Sensitivity: Applications in Blast, Shockwave, and Penetration Mechanics. Elsevier Science.

Plimpton, S., 1995. Fast parallel algorithms for short-range molecular dynamics. Journal of Computational Physics 117, 1-19.

Rinaldi, R. G., Boyce, M. C., Weigand, S. J., Londono, D. J., Guise, M. W., 2011. Microstructure evolution during tensile loading histories of a polyurea. Journal of Polymer Science Part B: Polymer Physics 49 (7), 1660-1671. 
Sinnokrot, M. O., Sherill, C. D., 2004. Highly accurate coupled cluster potential energy curves for the benzene dimer: Sandwich, t-shaped, and paralleldisplaced configurations. J. Phys. Chem. A 108, 10200-10207.

Voigt, W., 1889. Ueber die beziehung zwischen den beiden elasticittsconstanten isotroper krper. Annalen der Physik 274 (12), 573-587.

Yeoh, O. H., 1993. Some forms of the strain energy function for rubber. Rubber Chemistry and technology 66 (5), 754-771.

Yildirim, E., 2016. Effect of intersegmental interactions on the morphology of segmented polyurethanes with mixed soft segments: A coarse-grained simulation study. Polymer 90, 1873-2291.

Yilgör, I., Yilgör, E., Wilkes, G. L., 2014. Critical parameters in designing segmented polyurethanes and their effect on morphology and properties: A comprehensive review. Polymer 58, 1-36.

Yin, W., Venkitachalam, S., E., J., Staggs, S., Leventis, N., Lu, H., Rubenstein, D. A., 2010. Biocompatibility of surfactant-templated polyureananoencapsulated macroporous silica aerogels with plasma platelets and endothelial cells. Journal of Biomedical Materials Research Part A 4, 14311439. 


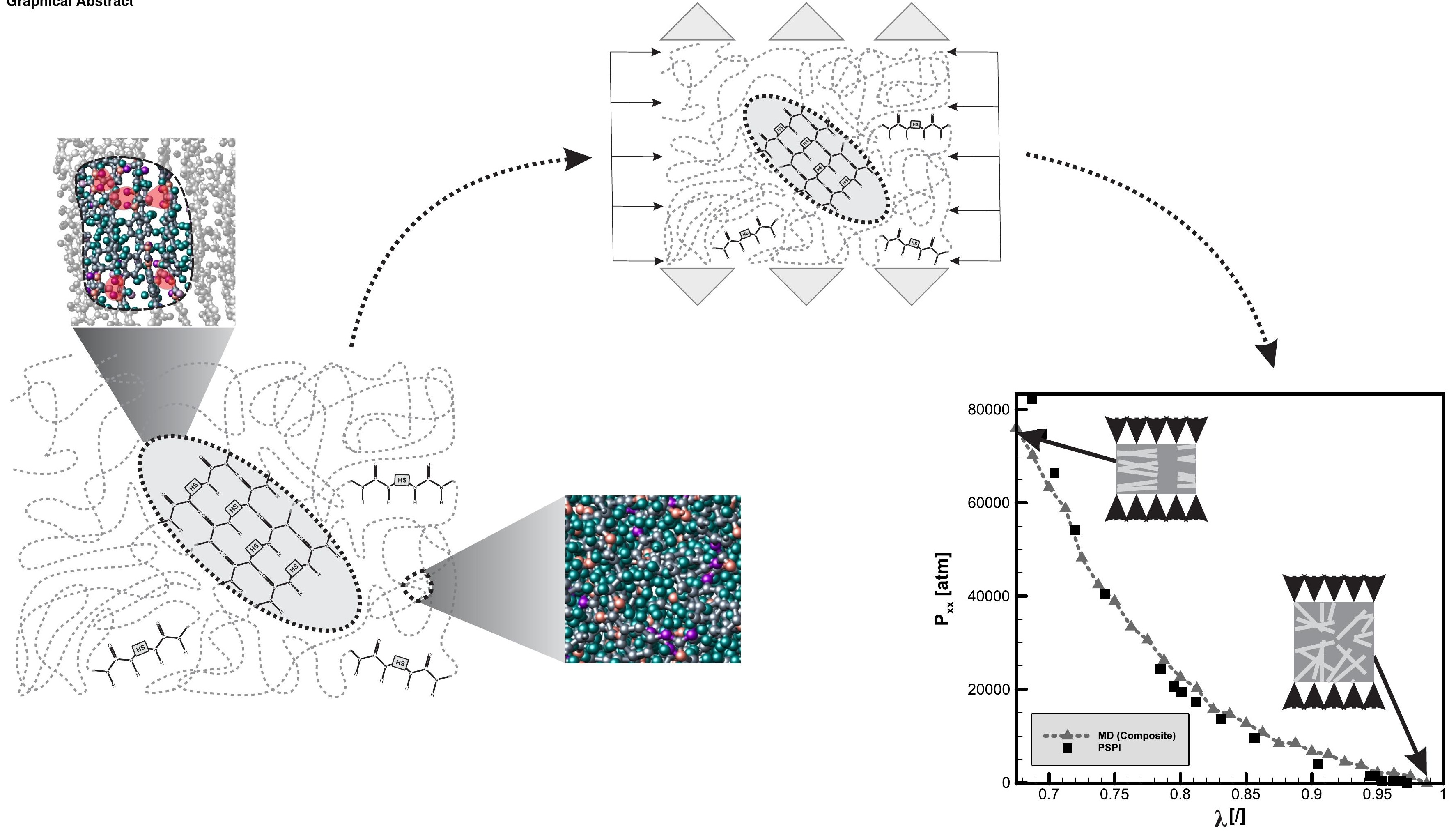

